\title{
Scope of Midwifery Practice: Concept Analysis
}

\author{
Abigail Kapfunde ${ }^{1}$, Clara Haruzivishe ${ }^{2}$, Christopher Samkange ${ }^{3}$, \\ Babill Stray-Pederson ${ }^{4}$ \\ ${ }^{1-3}$ University of Zimbabwe. College of Health Sciences. Department of Nursing Science. \\ ${ }^{4}$ University of Oslo, Institute of Clinical medicine. Division of Women and Children. Norway
}

\begin{abstract}
The objective of the paper was to describe the concept Scope of Midwifery practice (SOMP). Scope of midwifery practice remains dynamic and varies in many countries. It takes several forms ranging from listing of services, interventions given to women during pregnancy, labour and delivery to competencies expected of individual to practice. International confederation of Midwives (ICM) does not prescribe a fixed definition of the concept SOMP but emphasized that it should be built upon its international definition of the midwife. This position allows individual countries to come up with own variation. Existence of variations have compounded negatively on midwifery practice leading to skill loss in some cases and lack of confidence to practice to full capacity hence the need to describe the scope of midwifery practice by assigning measurable attributes for standardisation in midwifery practise. A literature search was conducted using PubMed, journal of midwifery and Medline which yielded 29 articles from 2006 to 2016. Filtering to consider only those describing the concept in relation to Midwifery yielded 6. The identified attributes defining the scope of midwifery practice were autonomy, leadership, competencies and practice setting (policy and regulations).
\end{abstract}

Keywords: Scope of Midwifery practice.

\section{Introduction}

The scope of practice remains a dynamic and variant concept in many professions. It can be considered as something internal not consciously considered yet others link it to competency, training or level of knowledge or professional conduct. [1] International Council Nursing (ICN) described the scope of practise for nurses as dynamic and responsive to health care needs. [2] In midwifery practice it may take several forms and should be in line with the international definition of the midwife. In view of the critical role played by the midwives in health service delivery it is important for each practitioner to understand their Scope of practice. ICM defined a midwife as a person who has successfully completed a midwifery education program which is duly recognised in their country and based on the ICM competences for basic midwifery practice as well as the ICM global standards for midwifery education, who acquired the requisite qualifications to be registered and or licenced to practice midwifery and use the title "Midwife", and who demonstrates competence in the practice of midwifery [3]. The midwife therefore is recognised as a responsible and accountable professional who works with and in partnership with women to give the necessary support, care and advice during pregnancy, labour and the postpartum period. This care includes preventive measures, the promotion of normal birth, detection of complications and emergency services. The role extends to counselling and education of women, family and community [3] Regulatory authorities mandated to define scope of midwifery practice should emphasize individual accountability, self-reliant, competence to enable practitioners to work to full scope of practice and meet consumer needs. A clearly defined scope of practice does not only improve patient outcomes but can lead to improved job satisfaction as well as positive migration along the competence continuum. On the contrary practitioners guided by an unclear scope of practice are more likely to take up roles or tasks beyond own competencies leading to increased patient risks and adverse outcomes [4]

\section{Materials And Methods}

An evolutionary approach was utilised as the concept of scope of midwifery practice and is dependent on the context and needs different parameters to appear in the scientific context to be interpreted. [5]A search was conducted using PubMed, Web of Science and MEDLINE. A preliminary search for terms scope of practice and Midwifery practice using Boolean terms "AND" and "OR" was conducted. To achieve a more precise description an inclusion and exclusion criteria was set. The inclusion criteria for final analysis were literature published in English from 2006 to 2016 in the context of midwifery practice. The year 2008 is when the ICM started developing the Global standards for midwifery regulation. The 2 years prior allowed for identification of the antecedents. Excluded were articles in non-English language and not related to midwifery practice. Preliminary search yielded 127 articles on Scope of practice and after inclusion criteria the number reduced to 29. In the final stage after deleting duplicated items (4) only 6 articles remained which had the definition of scope of midwifery and defining attributes of the concept. All articles in the context of nursing and midwifery were read, useful features, antecedents, consequences, related concepts and definition of the concept were extracted. Key characteristics of the concept were identified.

\begin{tabular}{lll|l}
\hline DOI: $10.9790 / 1959-0603033842$ & www.iosrjournals.org & $38 \mid$ Page
\end{tabular}




\section{Results}

The first stage of concept analysis is the Identification of the characteristics that leads to the definition of the concept [5] The defining characteristics identified were collaborative practice $[6 ; 7 ; 8]$ educational preparation, experience and competences $[3 ; 8]$, autonomy and accountability $[6 ; 9 ; 10 ; 11 ; 3]$, practice setting/policies and guidelines $[3 ; 12 ; 13 ; 14 ; 15]$ and lastly leadership and support. $(4 ; 16]$

\subsection{Defining characteristics of SOMP \\ 3.1.1 Collaborative Practice}

Midwives can realize their full SOP when they work collaboratively with other health professionals. They can consult, collaborate, delegate and communicate with different health care practitioners in their discharge of duties. The key aspects in collaborative practice is the relationship between professionals [6], professionals need to have an understanding and knowledge of the other's scope of practice as well as effective communication of actions $[7 ; 8]$. The Code of conduct should set this standard and support this important aspect of midwifery practice.

\subsubsection{Educational preparation, experience and competences}

The educational preparation of the midwives determines their SOP. The experience and the competence each individual practitioner has set the parameters of their practice. The midwife should make own judgement whether they or not competent to carry out a particular procedure. This is critical in determining one's SOP. ICM defines the midwife's educational preparation, experiences and basic essential competences for the individual prior to practice [3]. Each individual State will then set their specific requirements for registration and educational standards which suit their national needs. The midwife should have successfully completed a recognised and approved midwifery education programme in the country and should demonstrate and maintain competency in the practice of midwifery to use the title "registered midwife". [3]

Core competences for midwifery practices define the essential knowledge, skills and behaviours that all midwives must possess as stipulated in their midwifery definition. It is the midwife's sole responsibility to maintain and acquire additional competences as mandated by their regulatory authorities hence it becomes critical for them to be capable of assessing their own competence levels [8]

\subsubsection{Autonomy and Accountability}

Autonomy originates from a Greek word autos meaning 'self' and nemein or nomos" meaning to 'hold sway'. This is the origin of the concept of self-governance. This implies midwives determine and control the standards for education, regulation and practice. Autonomy is a concept central to the definition of midwife which means freedom to make choices of care and for the decision to be respected. $[4 ; 3 ; 12]$ This remains complex and the degree of autonomy demonstrated by midwives is variable and is dependent on the authority they are given in their work places as well as their willingness to accept the freedom. [9]

Midwives who are autonomous in thought are most likely to be capable of finding their way to provide women centred care regardless of the system or the working environment. Autonomous thinking may be influenced by training and supportive supervision while on the other hand autonomy in practice require more than independence of thought. A collaborative working relationship also fosters true autonomy as there is need for a viable backup system to own decisions for smooth transition in cases of emergency. [6] American heritage dictionary also defines an autonomous individual as one not controlled by other or outside forces, independent in judgement, self-directed, self-governing with or without respect to local or internal affairs.

Midwifery autonomy means having the ability to make some decisions within their profession and the right and responsibility to act according to shared standards. [11]. this implies having the competences to make the informed decision and the capacity to accept responsibility and be accountable for the outcomes of the decisions made. [3] Professional autonomy then refers to when there is ability to use various competences in a critical manner for safe quality health care to the patient $[10 ; 11]$.

\subsubsection{Practice setting, policies and Guidelines.}

A midwife can practice in any setting including in the home, in the community, in health institutions such as hospitals, clinics and maternity homes, [3]. Authority to practice is mandated by the individual society hence the primary motivation for practice should focus on patient's needs, safety and respect for their dignity. A good relationship with the patient is the fundamental aspect of midwifery practice as it fosters individual patient autonomy, informed choices and evidence based decision-making. In high risk or emergency situations the midwife's critical role in management focuses on assessing, collecting data and communicating it. They may have input in management in a collaborative pattern of care as they optimize normality. Clinical parameters are determined basically by the community in which one practices. This involves the lived experiences, the philosophy of the individual practitioner, community needs, nature of collaborative relationships and the 
practice guidelines of the area. Practice setting like SOP vary from place to place and with timing for example in central hospitals midwifery practice is limited to normal prenatal, intra and post-partum whereas out in the primary health care settings and communities the scope becomes broader to include managing complications, $[13 ; 14 ; 15]$. practice environment is largely regulated by state laws, policies and guidelines. Regulations are a set of criterion or processes arising from legislation and usually prescribed by the regulatory authority. State laws defining midwifery practice vary from state to state, national boards and national standards or codes of conduct. ICM has Code of ethics and code of conduct for midwifery professionals.

\subsubsection{Leadership and supervision}

Midwives need support systems for them to grow professionally and to recognise their full potential. Evidence has shown that nurses and midwives who practice without guidance and supportive leadership are likely to take responsibilities beyond own SOP and competences putting patient's lives at risk. [4]. Leadership and support supervision becomes critical in midwifery practice and competence development. A strong and supportive leadership play an important role in ensuring safe and effective care as well as boost confidence among practitioners, [16].

\section{Definitions}

The scope of midwifery practice is defined as the range of roles, functions, responsibilities and activities which a registered midwife is educated, competent and has the authority to perform in the context of the midwifery definition.[17;3]. The SOMP can also be defined as the extent or limits of intervention that a midwife can perform, $[18 ; 25]$. The attributes to the current definition is centred on education, competence and authority. There is need for one to be educated to practice midwifery. Another important dimension is the competence expected of the individual to be authorised to practice midwifery care. The apparent weakness with this definition is that education, competence and authority are dynamic variables which are not constant. The dynamic demographics of the community influence the scope of the practice. The defining attributes for the SOMP therefore should consider that the SOMP is an evolving process which is responsive to clinical service, demographic changes and fiscal changes of a given setting, [19; 26; 27]

\section{Discussion}

The evolutionary concept analysis involves identification of surrogate terms, related concepts, attributes, antecedents and consequences of the concept. Model cases or exemplars can also be used to clarify the concept.

\subsection{Surrogate and related concepts}

Surrogate terms are terms expressing the same meaning of the desired concept [20]. The terms which are used interchangeable for SOMP are range or extent of midwife's practice, professional regulation, roles and functions of a midwife $[21 ; 3 ; 22 ; 4 ; 23]$ Related terms refer to the terms used to describe concepts that are related to the desired concept but do not necessarily have similar characteristics. [5]. Related terms which are commonly used are legal parameters, boundaries, limits, competences, standards of practice, professional conduct $[3 ; 13 ; 14 ; 7 ; 8]$

\subsection{Antecedents of SOMP}

The next stage in the evolutionary concept analysis involves determination of the antecedents and consequences of the concept. Antecedents are defined as events that precedes occurrence of the event and consequences occur as a result of the antecedents. [5; 29] Identified antecedents fall under three groups: legal issues, economic issues and professional issues.

\subsubsection{Legal factors}

Fear of legal consequences, need to balance practise restrictions with practice expansion and right to practice within defined parameters $[13 ; 8 ; 9 ; 3]$. The need for and internationally accepted standards, policies and guidelines, code of conduct and the need to respond to the aesthetic aspect of patients' safety, needs and choices, regulatory requirements $[3 ; 24 ; 10 ; 11]$

\subsubsection{Economic factors}

Economic pressure, staff shortages, limited resources, time fiscal changes has led to variability of SOMP and expansion of practice. $[24 ; 28 ; 19 ; 23 ; 4 ; 30]$

\subsubsection{Professional factors}

Professional accountability, expectations around responsibility, perception and experience of practice expansion influence decision making. [8;19; 13] Professional autonomy, role confusion, lack of recognition, need for new reforms like continuity of care to childbearing women $[8 ; 19 ; 28 ; 1 ; 13 ; 25]$. Identified is also the need to develop and maintain competence, collaborative practice and educational preparation for safe and quality practice $[8 ; 1 ; 14 ; 3 ; 7 ; 22]$ 


\subsection{Consequences of the concept}

Scope of practice should provide a very public statement about the state of a profession in any given area hence an unclear definition may result in skill loss, deskilling, un-utilized staff potential and unmet patient needs, [13]. The midwife may suffer stagnation as they fear litigation if they expand practice or perceive they are encroaching into others territories. There is loss of confidence and no progress along the competence continuum. The end result is acceptance of reforms due to failure to understand own roles. This may consequently lead to powerlessness, inability to make professional decisions and accepting responsibility to act in accordance to shared standards. $[9 ; 10 ; 11]$

\subsection{Model case}

A model case is necessary to clarify the concept and delineate it from related concepts. After synthesis of literature model cases were identified to clarify defining attributes of SOMP.

Case 1: Chipo a 35 year old presents at a rural hospital with bleeding following a home delivery. She is pale, semi-conscious and Blood Pressure reading 70/30mmh.

Midwife A: at rural hospital where there is no doctor, no blood or blood products, no ambulance and laboratory to do cross match. The midwife does a rapid assessment excluded all causes of bleeding and identified retained placenta, collects blood specimens, put up 2 intravenous lines with plasma expanders to replace lost fluids, administer an oxytocic and try to remove retained placenta while awaiting ambulance to take her to next level. She explains condition to relative and continuously reassures patient that the personnel will do their best.

Midwife B: level 2 Hospital with all basic emergency facilities, laboratory, physicians, operating theatre and blood and blood products. She admits patient, checks vital signs and call doctor for management notifies laboratory and operating theatre of the emergency while continuously reassuring patient. She sets a trolley with intravenous insertion requirements, specimen collection tubes and gives an oxytocic intramuscularly.

The cases demonstrate the varying scopes of Midwifery practice. Midwife A has the competences to respond to an emergency though works within a limiting clinical parameters and is authorised to make decisions in response to patient's needs and consider patient safety. Practice parameters are influenced by availability of resources and patient needs. Midwife B follows guidelines and collaboratively manage the patient. Her practice is influenced by practice setting and limited authority. She cannot demonstrate her full competences. However the care received by the patient in both cases was within each midwife's SOP compatible with national standards.

\section{Working Definition}

The proposed definition of SOMP after appraisal of literature is:- the range of midwifery roles and responsibilities responsive to patient/community needs in different situations and practice settings as authorised for midwives according to nationally expected competences and professional standards. The SOMP though varying according to competence level, setting and country specific should be more consumers driven. That is it should respond to the individual patient/community needs.

\section{Relevancy To Clinical Practice}

Midwives play a critical role in maternal and child health therefore it is vital to have a clear definition of the concept to empower and enable them to practice to their full potential. The individual midwifes' understanding of SOMP fosters accountability and autonomy which positively impacts on the patient care outcomes and results in positive migration towards clinical competence continuum.

\section{Conclusion}

Scope of midwifery practice though an evolving concept emphasise the response to patient/community needs and should be flexible to suit all practice settings. The regulatory authorities should ensure that they review their standards to keep in line with dynamic needs of the patients and communities. Individual midwives also need to migrate positively with the continuum.

\section{Conflict of Interest}

The author has no conflict of interest.

\section{Reference List}

[1]. Casey, M., Fealy, G., Kennedy, C., Hegarty, J., Prizeman, G., McNamara, M. et al. (2015). Nurses', midwives' and key stakeholders' experiences and perceptions of a scope of nursing and midwifery practice framework. $J$ Adv.Nurs., 71, 1227-1237.

[2]. International Council of Nurses (2009) Nursing Matters: Nurse Practitioner/Advanced Practice Nurse: Definitions and Characteristics. Geneva, International Council of Nurses.

[3]. International Confederation of Midwives (2011) ICM International Definition of the Midwife. The Hague, International Confederation of Midwives. 
[4]. Lubbe J.C. \& Roetsl L. (2014) Nurses' scope of practice and the implication for quality nursing care. Journal of Nursing Scholarship 46(1), 58-64.

[5]. Rodgers BL, Knafl KA. Philadelphia: Saunders; 2000. Concept development in nursing: Foundations, techniques, and applications.

[6]. World Health Organization, (2010) Framework for Action on Interprofessional Education \& Collaborative Practice. Geneva, WHO, Department of Human Resources for Health.

[7]. Krautscheid, C. (2014) Defining Professional Nursing Accountability: A Literature Review Journal of Professional Nursing 30(1), 43-47.

[8]. Fealy, G., Casey, M., Rohde, D., Brady, A. M., Hegarty, J., Kennedy, C. et al. (2015). Scope of practice decision making: findings from a national survey of Irish nurses and midwives. J Clin.Nurs., 24, 2871-2880.

[9]. Starr, L. (2012). Policy, scope of practice and the 'agony of the moment'. Aust.Nurs.J, 19, 29.

[10]. Skar, R. (2009). The Meaning of Autonomy in Nursing Practice. Journal of Clinical Nursing 19, $2226-2234$.

[11]. Varjus, S. L., Leino-Kilpi H. and Suominen T. (2010) Professional autonomy of nurses in hospital settings - a review of the literature. Scandinavian Journal of Caring Sciences 25(1), 201-207.

[12]. International Confederation of Midwives (2014). Core Document, International code of ethics for midwives.

[13]. Sharma, B., Johansson, E., Prakasamma, M., Mavalankar, D., \& Christensson, K. (2013). Midwifery scope of practice among staff nurses: a grounded theory study in Gujarat, India. Midwifery, 29, 628-636.

[14]. Yang, Y. T., Attanasio, L. B., \& Kozhimannil, K. B. (2016). State Scope of Practice Laws, Nurse-Midwifery Workforce, and Childbirth Procedures and Outcomes. Womens Health Issues, 26, 262-267.

[15]. Weitz, T A, Taylor P, Diana et al (2009). Advancing Scope of Practice for advanced practice clinicians: more than a matter of access. Contraception, Vol 80, issue 2. 105-107.

[16]. National Clinical Effectiveness Committee (2013) National Clinical Effectiveness Committee: Guideline Developers Manual. Dublin, National Clinical Effectiveness Committee

[17]. European Commission (2005) European Parliament and Council Directive: The Professional Qualifications Directive (Directive 2005/36/EC). Brussels, European Commission.

[18]. Mosby's Medical Dictionary (2009), Mosby/Elsevier. ISBN 0323052908, 9780323052900

[19]. Kennedy, C., O'Reilly, P., Fealy, G., Casey, M., Brady, A. M., McNamara, M. et al. (2015). Comparative analysis of nursing and midwifery regulatory and professional bodies' scope of practice and associated decision-making frameworks: a discussion paper. $J$ Adv.Nurs., 71, 1797-1811.

[20]. Rodgers BL. 2nd ed. Saskatchewan, US: W.b. Saunders; 2000. Concept development in nursing: Foundations, techniques, and applications; pp. 77-102.

[21]. American Academy of Nurse Practitioners. (2011). Scope of practice for nurse practitioners, 2010. Retrieved June 15, 2011, from http://www.aanp.org/NR/rdonlyres/FCA07860-3DA1-46F9-80E6-E93A0972FB0D/0/2010ScopeOfPractice.pdf

[22]. Bigham B.L., Kennedy S.M., Drennan I. \& Morrison L.J. (2013) Expanding paramedic scope of practice in the community: a systematic review of literature. Prehospital Emergency Care 17(3), 361-372.

[23]. Goemaes, R, Beeckman, D, Goossens, J, Shawe, JA, Verhaeghe, S and Van Hecke, A (2016) Advanced midwifery practice: An evolutionary concept analysis Midwifery, 42. pp. 29-37.

[24]. Sidebotham, M., Fenwick, J., Rath, S., \& Gamble, J. (2015). Midwives' perceptions of their role within the context of maternity service reform: An Appreciative Inquiry. Women Birth, 28, 112-120.

[25]. Homer, C. S., Passant, L., Kildea, S., Pincombe, J., Thorogood, C., Leap, N. et al. (2007). The development of national competency standards for the midwife in Australia. Midwifery, 23, 350-360.

[26]. Jacob, E. R., Barnett, A., Sellick, K., \& McKenna, L. (2013). Scope of practice for Australian enrolled nurses: evolution and practice issues. Contemp.Nurse, 45, 155-163.

[27]. Fox-Young, S. \& Ashley, C. (2010). Developing an Australian framework for scope of practice decisions by nurses and midwives lessons for cross-border standards development. J Clin.Nurs., 19, 2235-2241.

[28]. Sidebotham, M. (2012). Responding to the changing landscape: Australian midwifery. Pract.Midwife., 15, 24-26.

[29]. Schluter J, Seaton P \& Chaboyer W (2011) Understanding nursing scope of practice: a qualitative study. International Journal of Nursing Studies 48, 1211-1222. 